\title{
Halo Shapes, Dynamics and Environment
}

\author{
Manolis Plionis ${ }^{1}$, Cinthia Ragone ${ }^{2}$ and Spyros Basilakos ${ }^{3}$ \\ 1 Institute of Astronomy \& Astrophysics, NOA, Athens, Greece \& INAOE, \\ Puebla, México \\ 2 IATE, Observatorio Astronómico, Córdova, Argentina \\ ${ }^{3}$ Kapteyn Institute, University of Groningen, the Netherlands
}

Summary. In the hierarchical structure formation model cosmic halos are supposed to form by accretion of smaller units along anisotropic direction, defined by largescale filamentary structures. After the epoch of mass aggregation (which depend on the cosmological model), violent relaxation processes will tend to alter the halo phase-space configuration producing quasi-spherical halos with a relatively smooth density profiles.

Here we attempt to investigate the relation between halos shapes, their environment and their dynamical state. To this end we have run a large $\left(L=500 \mathrm{~h}^{-1}\right.$ Mpc, $N_{p}=512^{3}$ particles) N-body simulation of a flat low-density cold dark matter model with a matter density $\Omega_{\mathrm{m}}=1-\Omega_{\Lambda}=0.3$, Hubble constant $H_{\circ}=70$ $\mathrm{km} \mathrm{s}^{-1} \mathrm{Mpc}^{-1}$ and a normalization parameter of $\sigma_{8}=0.9$. The particle mass is $m_{\mathrm{p}} \geq 7.7 \times 10^{10} h^{-1} M_{\odot}$ comparable to the mass of one single galaxy. The halos are defined using a friends-of-friend algorithm with a linking length given by $l=0.17 \bar{\nu}$ where $\bar{\nu}$ is the mean density. This linking length corresponds to an overdensity $\rho / \rho_{\text {mean }} \simeq 200$ at the present epoch $(z=0)$ and the total number of halos with more than 130 particles $\left(M>3 \times 10^{13} h^{-1} M_{\odot}\right)$ is 57524 .

\section{Halo Shapes and Environment}

Halo shapes are defined by diagonalizing the moments of inertia from which we derive the eigenvalues and eigenvectors of the best ellipsoidal halo fit. The principal axes $a, b, c$ are related to the square root of the eigenvalues such that $a>b>c$. Our results, in agreement with other studies, indicate that although halos are triaxial they are significantly more prolate than oblate. This is quantified by using the so called triaxiality index [5] defined as $T=$ $\left(a^{2}-b^{2}\right) /\left(a^{2}-c^{2}\right)$, which has limiting values of $T=1$ and $T=0$ for the case of a pure prolate and oblate spheroid, respectively. Our results show that the fraction of halos with pronounced prolatness (ie., large $T \mathrm{~s}$ ) is significantly higher than that of oblate-like halos. Overall we obtain from our simulated halos that $\langle T\rangle \simeq 0.73$. 
An interesting question, especially for observational studies, is whether the $3 \mathrm{D}$ halo shape distribution can be inferred from the projected (2D) shapes. This is an inversion problem for which, under the assumption of random halo orientation with respect to the line of sight and purely oblate or prolate spheroidal halos, there is a unique relation between the projected and intrinsic axial ratio distributions. This is described by a set of integral equations, first investigated by [6] and given by [15]:

$$
\hat{f}(q)=\frac{1}{q^{2}} \int_{0}^{q} \frac{\beta^{2} \hat{N}_{p}(\beta) \mathrm{d} \beta}{\left(1-q^{2}\right)^{1 / 2}\left(q^{2}-\beta^{2}\right)^{1 / 2}}, \hat{f}(q)=q \int_{0}^{q} \frac{\hat{N}_{\circ}(\beta) \mathrm{d} \beta}{\left(1-q^{2}\right)^{1 / 2}\left(q^{2}-\beta^{2}\right)^{1 / 2}}
$$

with $\beta$ representing the intrinsic axial ratio while $\hat{N}_{o}(\beta)$ and $\hat{N}_{p}(\beta)$ the intrinsic prolate and oblate axial ratio distributions, respectively. The continuous function $f(q)$ is derived from the discrete axial ratios frequency distribution using the so-called kernel estimators (for details see [14] and references therein). Inverting then the above equations gives us the distribution of true axial ratios as a function of $f(q)$ (eg. [4]). Nevertheless, if halos are a mixture of the two spheroidal populations or they have triaxial configurations there is no unique inversion [12]. However, all may not be lost and although the exact shape distribution may not be recovered accurately one could possibly infer whether the $3 \mathrm{D}$ halo shapes are predominantly more prolate or oblate-like. Let us see this in more detail using our simulated intrinsically triaxial ellipsoidal halos which are however dominated by prolate-like shapes $(\langle T\rangle \simeq 0.73)$. The important point here is that in order for the inverted distribution to be physically meaningful it should be positive for all $\beta$ 's. Negative values indicate that the assumed model for the intrinsic halo shape is unacceptable.

In Fig.1 (left panel) we present the discrete and continuous - $f(q)$ - distributions of the projected in 2D axial ratios for halos of two mass ranges (indicated in the plot). In the middle and right panels we present the inverted $3 \mathrm{D}$ axial ratio distributions (continuous lines) for the prolate and oblate models, respectively. It is evident that the inverted oblate-model distribution has many negative values which is an important indication that this model is unacceptable, while the opposite is true for the prolate-model distribution. Furthermore, we plot as histograms the intrinsic axial ratio distribution of the "average" prolate or oblate spheroidal fit to the $3 \mathrm{D}$ halo. These fits are realized by estimating the corresponding axial ratios by $\beta_{\mathrm{P}}=(b+c) / 2 a$ and $\beta_{\mathrm{O}}=2 c /(a+b)$. It is evident that the purely oblate model fails miserably to even come close to the inverted distribution while the prolate model fits relatively well the corresponding inverted 3D prolate-model distribution. This agrees with the higher prolatness of the 3D halo shapes. We therefore conclude that applying the previously discussed inversion method to observational data (eg. [13], [11]) we can infer, even in the event of triaxial ellipsoidal halo shapes, the dominance of prolate or oblate-like 3D shapes.

Another interesting fact, shown in recent high-resolution simulations of the concordance model, is the correlation between halo mass and halo flattening, 


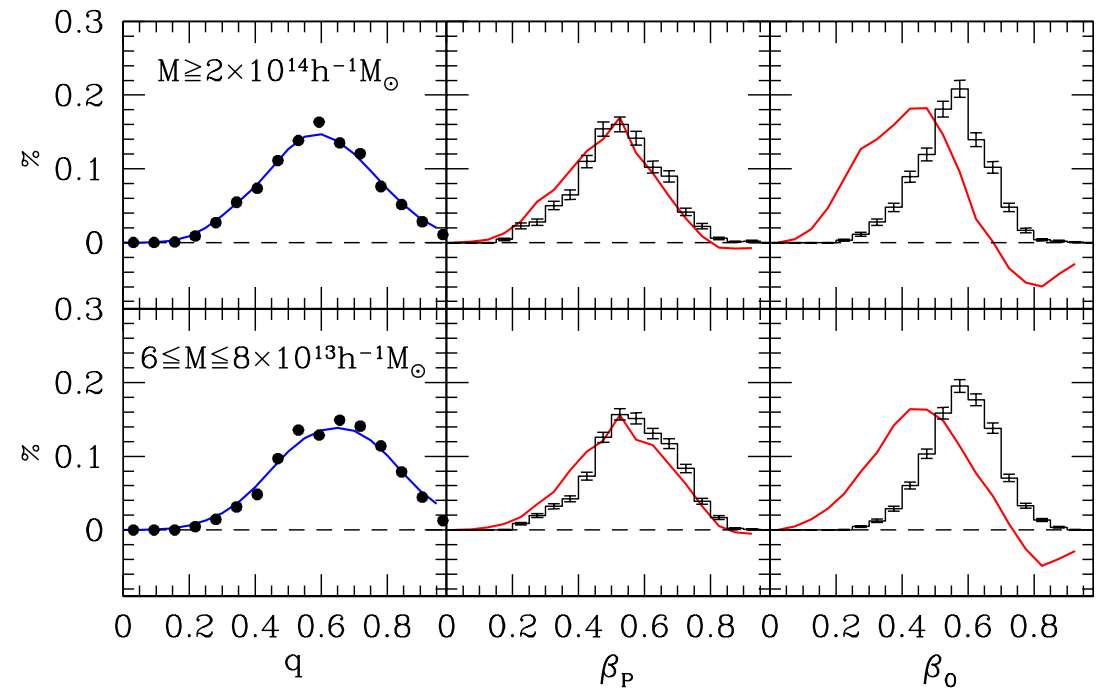

Fig. 1. Left Panel: The projected axial ratio distributions for two different halo mass thresholds with the nonparametric kernel estimator fit (solid line). Central Panel: The inverted intrinsic halo axial ratio distribution (continuous line) for the prolate model together with distribution of "average" prolate spheroidal fit to the 3D halos. Right Panel: the corresponding distributions for the oblate case.

with more massive halos being flatter (eg. [1], [8], [7]). This is counter-intuitive in the sense that the massive halos should collapse faster than lower mass halos of roughly the same formation age and thus they should have more time to dynamically evolve and virialize, a process that should reduce their initial ellipsoidal configuration. However, halo formation ages vary and secondary infall, which if important it could affect the halo outskirts, can produce such elongated halo geometry. However, even in such a case the inner parts of the most massive halos should be virialized and thus nearly spherical, which however does not seem to be the case (eg. [1] their figure 7). Note that these results are based on dissipationless simulations while baryonic dissipation has been shown to affect halo shapes, producing significantly rounder halos (eg. [9]). Also one may expect that halos in low-density regions, where tidal effects, accretion and merging are minimal to be less elongated, as indeed has been found (eg. [2]).

\section{Halo Dynamics \& Environment}

In order to define the dynamical state of a halo we use the $\Delta$-deviation substructure statistic [3], which looks for deviations of the local velocity mean 


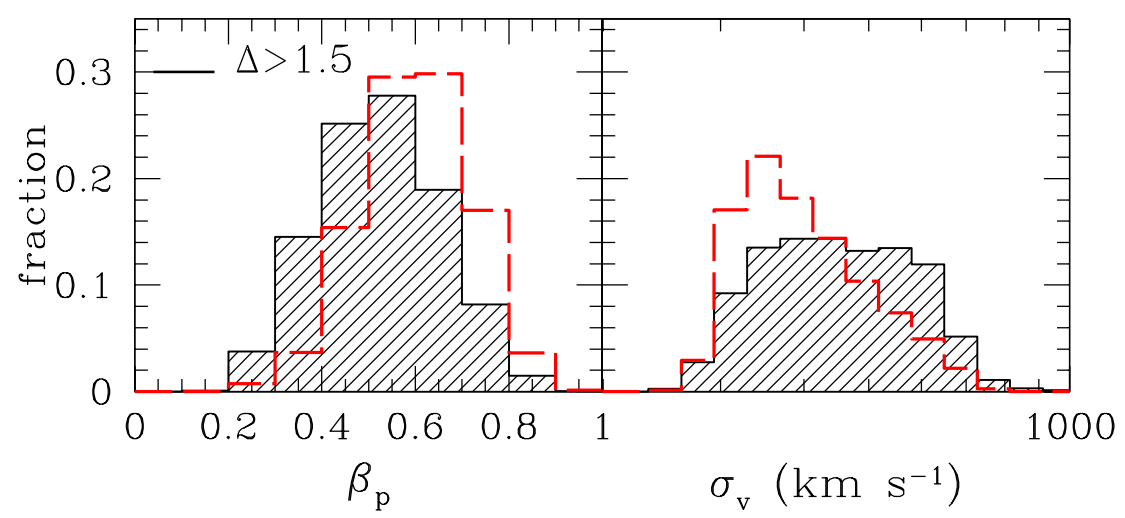

Fig. 2. Normalized axial ratio and velocity dispersion distributions. Hatched histograms represent halos with significant substructure while the dashed-line histograms represent the overall halo population.

and dispersion around their overall halo values. For each halo particle we find its $N_{n n}$ nearest neighbors from which we calculate their local velocity mean and dispersion which we then compare with their overall halo values. The local deviations are defined by: $\delta_{i}^{2}=\frac{N_{n n}}{\sigma_{v}^{2}}\left[\left(\bar{v}_{\text {local }}-\bar{v}\right)^{2}+\left(\sigma_{v, \text { local }}-\sigma_{v}\right)^{2}\right]$ while the individual halo $\Delta$-statistic is given by the sum $\Delta=\sum \delta_{i}$. It has been found that a robust measure of the substructure index is given when using $N_{n n}=25$ [10], which is the value that we use. In figure 2 we show the velocity dispersion (right panel) and $\beta_{P}$ axial ratio (left panel) distributions for halos in the mass range $3 \times 10^{13}<M<8 \times 10^{13} h^{-1} M_{\odot}$. Those that have a substructure index $\Delta>1.5$ are shown as hatched histograms while the overall halo distributions as dashed-line histograms. It is evident that dynamically young halos (ie., those with significant substructure) are more elongated and have a higher velocity dispersion than the overall halo population.

Furthermore, we investigate the correlation between halo velocity dispersion and halo mass. From our halo identification procedure we expect that most halos should be nearly virialized and thus their velocity dispersion should be strongly correlated with their mass via the virial theorem. Indeed, there is a strong correlation between the velocity dispersion and the halo mass, measured by summing the member DM particles masses, with a Spearman correlation coefficient of $R \sim 0.9$. We find however, that this strong correlation breaks down in the vicinity of large halo hosts. In figure 3 we show with open points the correlation coefficient between halo $\sigma_{v}$ and Mass as a function of distance to their nearest massive $\left(M>2 \times 10^{14} h^{-1} M_{\odot}\right)$ host neighbor. The monotonic drop of the correlation coefficient with decreasing halo-host separation is evident. We also find that there is a significant although weaker correlation between $\sigma_{v}$ and the substructure index $(\Delta)$, which increases as the 


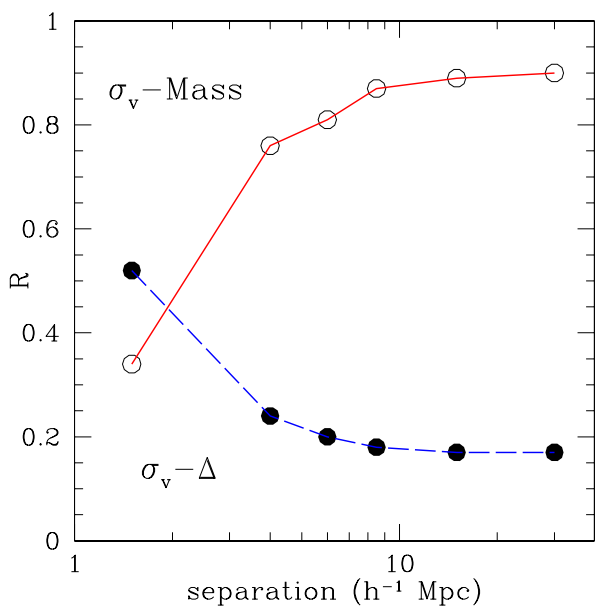

Fig. 3. Spearman correlation coefficient for the $\sigma_{v}$ vs Mass and $\sigma_{v}$ vs $\Delta$ correlations as a function of host-halo distance.

halo-host distance decreases. This probably implies that halos near large hosts are either disrupted due to the presence of strong tidal fields or more probably that the excess density of sub-halos near hosts induce strong inter-halo gravitational interactions and merging. These results have important consequences for observational studies and put important limits on the validity of using the virial theorem to estimate group or cluster masses in the vicinity of massive clusters.

\section{References}

1. Allgood et al., ApJ, in press, astro-ph/0508497

2. Avila-Reese, V., et al., ApJ, 634, 51 (2005)

3. Dressler, A. \& Shectman, S.A., AJ, 95, 985 (1988)

4. Fall, M. \& Frenk, C. S., ApJ, 88, 1626 (1983)

5. Franx, M., Illingworth, G., de Zeeuw, T., ApJ, 383, 112 (1991)

6. Hubble, E.P., ApJ, 64, 321 (1926)

7. Jing, Y.P. \& Suto, Y., ApJ, 529, L69 (2002)

8. Kasun, S.F. \& Evrard, A.E., ApJ, 629, 781 (2005)

9. Kazantzidis, S. et al., ApJ, 611, L73 (2004)

10. Knebe, A., Müller, V., A\&A, 354, 761 (2000)

11. Paz, D.J., Lambas, D.G., Padilla, N., Merchan, M., MNRAS, in press (2006)

12. Plionis M., Barrow J.D., Frenk, C.S., MNRAS, 249, 662 (1991)

13. Plionis M., Basilakos, S., Tovmassian, H., MNRAS, 352, 1323 (2004)

14. Ryden, B.S., ApJ, 461, 146 (1996)

15. Sandage, A., Freeman, K.C. \& Stokes, N.R., ApJ, 160, 831 (1970) 